\title{
Use of totally implantable central venous access port via the basilic vein in patients with thoracic malignancies.
}

\section{$\operatorname{AUTHOR}(S)$ :}

Sonobe, Makoto; Chen, Fengshi; Fujinaga, Takuji; Sato, Kiyoshi; Shoji, Tsuyoshi; Sakai, Hiroaki; Miyahara, Ryo; ... Okubo, Kenichi; Hirata, Toshiki; Date, Hiroshi

\section{CITATION:}

Sonobe, Makoto ... [et al]. Use of totally implantable central venous access port via the basilic vein in patients with thoracic malignancies.. International journal of clinical oncology / Japan Society of Clinical Oncology 2009, 14(3): 208-212

\section{ISSUE DATE:}

2009-06

URL:

http://hdl.handle.net/2433/87298

\section{RIGHT:}

c The Japan Society of Clinical Oncology 2009.; This is not the published version. Please cite only the published version.; この論文は 出版社版でありません。引用の際には出版社版をご確認ご利用くださ し。 
Title:

Use of totally implantable central venous access port via the basilic vein in patients with thoracic malignancies

\section{Authors:}

Makoto Sonobe, Fengshi Chen, Takuji Fujinaga, Kiyoshi Sato, Tsuyoshi Shoji, Hiroaki Sakai, Ryo Miyahara, Toru Bando, Kenichi Okubo, Toshiki Hirata, Hiroshi Date

\section{Institution:}

Department of Thoracic Surgery, Kyoto University Hospital

\section{Address:}

Shogoin-kawaracho 54,

Sakyo-ku,

Kyoto 606-8507, Japan

Tel: 81-75-751-4975

Fax: 81-75-751-4974

E-mail address of first author: mysonobe@kuhp.kyoto-u.ac.jp

\section{Corresponding to:}

Makoto Sonobe,

Department of Thoracic Surgery, Kyoto University Hospital

Shogoin-kawaracho 54, Sakyo-ku,

Kyoto 606-8507, Japan

Tel: 81-75-751-4975, Fax: 81-75-751-4974

E-mail address: mysonobe@kuhp.kyoto-u.ac.jp 


\section{Abstract}

Background: For patients with a thoracic malignancy whose peripheral veins are not suitable for blood access for chemotherapy, we evaluated a totally implantable central venous access port, in which the port is implanted in the ulnar side of arm and the catheter is introduced via the basilic vein into the superior vena cava (TIAP-BV).

Methods: Twenty-five patients (21 with lung cancer, 2 with malignant pleural mesothelioma, and 2 with thymoma) receiving TIAP-BV were included. Indication, surgical complications, and long-term complications were analyzed.

Results: Indications for TIAP-BV were: chemotherapy (17 patients); chemotherapy with parenteral nutrition (8 patients). The following surgical complications occurred: arrhythmia due to misplacement of the tip of catheter (1 patient); intraoperative conversion from the left to right arm (2 patients); hematoma at the implantation site (1 patient). Short-term problems were: death 1 week after implantation without the use of TIAP-BV (2 patients). Long-term complications were: skin ulcer at the port site (1 patient); early removal of TIAP-BV because of port site infection (1 patient); catheter occlusion (1 patient); venous thrombosis of basilic vein (2 patients). In the 22 patients who did not die early or have the device withdrawn early, the median duration of TIAP-BV use was 7 months (range, 1 to 20) without any break to the port system, leakage of drugs, or catheter-related infections. 
Conclusion: TIAP-BV can endure the long-term use for chemotherapy and

parenteral nutrition. However, a patient's expected prognosis and infectious disease status at time of implantation surgery should be considered before surgery proceeds.

\section{Mini-abstract}

We present on the safety of the implantation and use of implantable venous port via the basilic vein in chemotherapy and parenteral nutrition for patients with thoracic malignancy.

\section{Key Word}

Totally implantable central venous access port, basilic vein, lung cancer 


\section{Text}

\section{Introduction}

In lung cancer chemotherapy, third-generation chemotherapeutic drugs

(paclitaxel, docetaxel, gemcitabine, vinorelbine, and irinotecan) have been associated with significant advances in survival benefit and improvement in the quality of life ${ }^{1}$, with platinum-based regimens the first choice. Third-generation monotherapy, as well as new drugs such as amrubicin or pemetrexate, are considered good candidates for second / third-line regimens ${ }^{2}$; in malignant pleural mesothelioma and invasive thymoma, several complex but effective regimens have been reported ${ }^{3,4}$. Therefore, patients with advanced thoracic malignancy frequently require repeated puncture of peripheral veins for blood tests, administration of consecutive chemotherapeutic agents, fluid administration, and occasionally parenteral nutrition over a long period of time.

During repeated puncture of peripheral veins, oncology practitioners sometimes encounter patients with peripheral veins no longer able to accept an indwelling needle. A consequence of this is that medical oncologists will not be able to find a reliable peripheral vein for administering the chemotherapeutic drugs that have a high potential for the induction of skin necrosis due to extravasation, such as vinorelbine ${ }^{5}$, docetaxel, paclitaxel, and amrubicin. 
For easy, safe and repeated venous access, totally implantable central venous access ports (TIAP), are widely used for the safe administration of chemotherapeutic drugs, blood transfusion and parenteral nutrition in oncology; these devices can also facilitate the problem of the venous access in thoracic malignancies ${ }^{6}$

For the placement of a TIAP, catheter introduction through the subclavian vein to the superior vena cava (SVC) via the percutaneous approach, is the most common technique ${ }^{6,7}$. However, this approach via the subclavian vein is associated with considerable complications including pneumothorax, hemothorax, injury of large vessels, and catheter pinch-off within the costclavicular space ${ }^{6-8}$. The venous cut-down approach through the external jugular vein or cephalic vein has been reported to be one of a number of safe and easy alternatives ${ }^{9-11}$.

In our department, prior to May 2006, we used the 'TIAP via subclavian vein' approach. However, with this method, we experienced two cases of pinch-off syndrome of the implanted catheter. From June 2006, therefore, we have used the TIAP with the port implanted in the ulnar side of the arm, and the catheter introduced via the basilic vein to the SVC (TIAP-BV), using the Seldinger method. We made this switch due to the easier technique for implantation compared to the cut-down approach, and the avoidance of pinch-off of the catheter. Herein, we report our 
experiences and problems associated with the use of TIAP-BV.

\section{Patients and methods}

\section{Patients}

This was a retrospective study in 25 patients with thoracic malignancy; all patients had peripheral veins that were too fragile for practitioners to insert the usual indwelling needles for chemotherapy and/or fluid administration. Between June 2006 and December 2007, these 25 patients underwent implantation of TIAP-BV in which the port was implanted in the ulnar side of arm and the catheter was introduced via the basilic vein to the SVC, because of the fragility of their peripheral veins.

\section{Implantation procedures of TIAP-BV}

Implantation surgery was performed at the outpatient surgery unit of our hospital. Prior to the procedure, the direction of the basilic vein in the ulnar side of the arm (usually the left one) was established using radiographic or ultrasonographic guidance (Figure 1). For left-handed patients, the right arm was selected. Under local anesthesia, an approximately $4 \mathrm{~cm}$ long incision was made in the skin in the center of the ulnar side of arm and a subcutaneous pocket was prepared for the port body (Vital-Port MINI®, IP-S5116, Cook Vascular Incorporated, Vandergrift, PA, USA) (Figure 2). The direction of the basilic vein was identified again during the operation 
under venography. According to the Seldinger method, the basilic vein was punctured with 20 gauge indwelling needle under venograpy (Figure 3A and 3B). And then a guide wire (Radifocus Guidewire M®, RF-GA25123, Terumo Co. Ltd, Tokyo, Japan) was introduced through the basilic vein (Figure $3 \mathrm{C}$ ) to the SVC. After dilating the puncture point of basilic vein with the introducer, a catheter was introduced along the guide wire and the tip of the catheter was placed in the SVC under radiographic guidance. The distal end of catheter was attached to the port body and the port was placed in the pocket. Flushing and filling with heparinized saline $(100 \mathrm{IU} / \mathrm{ml})$ was performed to verify the flow through the port system.

\section{Use of TIAP-BV}

If no incidences of hematoma, infection, or serous fluid retention were identified during a period of 3 to 7 days after implantation, then the TIAP-BV was used. The medical staff who used the TIAP-BV were all well-trained staff physicians of the Outpatient Oncology Unit or the Department of Respiratory Medicine, or staff surgeons of the Department of Thoracic Surgery at our institute. For puncture of the port, a 22-gauge non-coring needle (Coreless Needle Set ${ }^{\circledR}$, Nipro Co. Ltd, Osaka, Japan) was used under aseptic conditions. After the TIAP-BV was used, it was flushed with $10 \mathrm{ml}$ of heparinized saline $(100 \mathrm{IU} / \mathrm{ml})$. In patients with a temporary interruption of their chemotherapy, heparin flushing of the TIAP-BV was performed at 
intervals of approximately 4-week intervals.

\section{Evaluation}

The reason for the implantation of the TIAP-BV, the number of regimens and cycles of chemotherapy before and after implantation, the operation time, complications of implantation surgery, usage of the TIAP-BV, and long-term complications / problems that occurred during the period from the implantation to April 2008, were all evaluated. Continuous variables were expressed as medians and the range given.

\section{Results}

Patient demographics and baseline characteristics are presented in Table 1.

Of 25 patients, 14 were male and 11 were female; they had a median age of 66 years (range, 39 - 79 years). Twenty-one patients had lung cancer, two had malignant pleural mesothelioma, and two had invasive thymoma.

The reasons for the use of TIAP-BV were: chemotherapy (17 patients) and chemotherapy with parenteral nutrition (8 patients). The median duration from the beginning of chemotherapy to implantation of TIAP-BV was 18 months (range, 0 to 84 months). The median number of chemotherapeutic regimens and cycles 
prior to TIAP-BV implantation was 2 (range, 0 to 11) and 8 (range, 0 to 46), respectively.

We planned initially to place the TIAP-BV on the left side in 24 patients, and on the right side in one. A left-sided placement was successful in 22 of the 24 patients as planned; in the other 2 patients it became necessary to convert to the right because the left basilic vein was too fragile to insert a dilating introducer. The right-sided placement was also successful in the one patient in whom the intention had always been to opt for this side. The median time for the implantation was 37 minutes (range, 27 to 120 minutes).

With respect of surgical complications (Table 2), arrhythmia (frequent paroxysmal atrial contraction) occurred in 1 patient, probably as a consequence of the tip of catheter touching the right atrium. After replacing the catheter tip on the SVC, the arrhythmia disappeared. In addition, a hematoma at the implanted site was reported in another patient, which resolved within 2 weeks. With respect of immediate mortality and morbidity problems (Table 2), 2 patients (8\%) died, within 1 week of implantation, before a TIAP-BV had been used, because of a rapid disease progression. In 1 (4\%) other patient, early removal of the TIAP-BV occurred, 8 days after implantation because of a port site infection, secondary to aspiration pneumonia with bacteremia due to methicillin-resistant Staphylococcus aureus. 
With respect of long-term complications (Table 2), venous thrombosis of the basilic vein in which the catheter was placed was observed in $2(8 \%)$ patients, approximately 3 weeks after implantation. These 2 patients received warfarin treatment and their TIAP-BV continued to be used without further complications, including pulmonary thromboembolism. Skin ulcer at the port site due to cutting of skin with needle during repeated puncture was observed in one patient (4\%) after 7 months of use for chemotherapy and parenteral nutrition. Port occlusion was documented in one patient (4\%) after 5 months of use for chemotherapy and parenteral nutrition. Among the 22 patients who were free of short-term complications (ie early death or early removal of the TIAP-BV), the TIAP-BV were used for a median of 7 months (range, 1 to 20 months) without damage to the port or catheter, leakage of drugs outside of the port system, pinch-off syndrome, or catheter-related infections. Of these 22 patients, 3 received none of their planned intravenous chemotherapy, but their TIAP-BVs were effectively used for parenteral nutrition or fluid administration. In the other 19 patients, the median number of chemotherapeutic regimens and cycles after TIAP-BV implantation was 2 (range, 1 to 3) and 9 (range, 1 to 21), respectively. As of April 2008, 10 out of 25 patients (40\%) had died since the placement of a TIAP-BV. 


\section{Discussion}

In this paper, we evaluated the experiences and the problems in the use of TIAP-BV in thoracic malignancy, because we had been aware that there were very few reports on the use of TIAP in the Japanese population or regarding the implantation approach through the basilic vein with the Seldinger method.

In our patient series, the median of chemotherapeutic regimens prior to TIAP-BV implantation was 2, consisting of 8 cycles in median. Currently, there is no definitive indication for timing the employment of TIAP in the treatment of thoracic malignancies. In our institute, docetaxel- or vinorelbine-containing regimens are frequently used as second-line regimens for lung cancer and the peripheral veins of patients occasionally become fragile during second-line chemotherapy. In this situation, subsequent administration of third-line chemotherapy containing irinotecan, gemcitabine, or amrubicin through the peripheral veins carries a high risk for the development of skin ulcers and necrosis due to extravasation of drugs. Therefore, maybe the beginning of third-line regimen with potentially necrosis-inducing agents would be timely for the placement of TIAP.

With respect to surgical complications, we experienced two cases requiring intraoperative conversion from the left to right basilic vein. In both cases, we had ascertained a good flow through the left basilic vein immediately before surgery using 
venography or ultrasonography. However, intra-operation findings indicated that the walls of the basilic veins in these 2 patients were very fragile and considerable blood leakage occurred at the point of puncture for the wire guiding. Therefore, we decided to switch to the right arm. Conversion to the cut-down method involving the cephalic vein at a more central portion such as the delto-pectoral groove may be good alternative $^{9}$ in these situations.

Arrhythmia and hematoma were also documented as surgical complications in our patient series. These would appear to be avoidable with careful catheter placement or hemostasis ascertainment.

Two early deaths were observed after implantation of TIAP-BV; both deaths were unrelated to TIAP-BV implantation, and due to disease progression. Furthermore, in neither patient had the TIAP-BVs been used for chemotherapy or fluid administration prior to death. Sadly, this means that for these 2 patients the implantation surgery had been in vain. This highlights the need for a careful evaluation of the prognosis of all potential candidates for TIAP-BV, prior to the procedure being started.

Catheter-related Infection is one important potential complication in the long-term management of TIAP. In the literature, infections related to totally implantable port systems occurred in $2.0 \%$ to $9.1 \%$ of patients ${ }^{12-15}$. In our series, 
although no catheter-related infections in the long-term management of TIAP-BV

were observed, one early port site infection due to bacteremia from aspiration pneumonia occurred immediately after implantation surgery was documented. This patient was aspiration-prone and easily developed bacteremia such as the one that caused this episode of catheter and port infection. Because the hematogenous infection of TIAP cannot be avoided, even with the strictest antiseptic procedures being followed during the puncture of the port, severely compromised patients including aspiration-prone patients or patients with an active infection should not be considered for implantation.

Thromboembolic complication is another important potential problem in the long-term management of TIAP. Catheter implantation itself carries a risk for venous thrombosis and cancer patients who need a TIAP have an increased risk of venous thrombosis ${ }^{6,16}$. Although the detailed incidence of thromboembolic complications in TIAP-BV that we use is unclear, Kuriakose et al. reported that a higher incidence of arm venous thrombosis in patients with a peripherally placed port (11.4\%) than with a chest port $(4.8 \%)^{17}$. The incidence of arm venous thrombosis in our series (8\%) is compatible to that of peripherally placed port. There are currently no definite recommendations or guidelines for the use of prophylactic anticoagulation therapy to prevent thromboembolic complications regarding TIAP ${ }^{6}$. Therefore, we feel that the 
prompt use of anticoagulation therapy after the occurrence of venous thrombosis is essential $^{16}$.

No complications regarding a breakdown of the port system or drug leaks were observed over the period of 1 to 20 months used in our series. This is probably because the main users of TIAP-BV at our institute were all highly trained in the use of several types of TIAP systems, including TIAP-BV, and thereby mechanical damage to the port system could be avoided. Like other types of TIAP, TIAP-BV can provide safe venous access for long-term use when used appropriately by trained practitioners.

In conclusion, for patients with peripheral veins no longer able to accept an indwelling needle, TIAP-BV is safe and suitable for the long-term use of chemotherapy, fluid administration, and parenteral nutrition in thoracic malignancy. Even from our limited experience, however, early death of patients and early removal after implantation are the most problematic waste use in application of TIAP-BV. Therefore, in considering the indication for the procedure, we should verify that a candidate of TIAP-PV has good performance status and organ functions enough to receive chemotherapy, has a certain level of prognosis when the chemotherapy would be effective, and has no active infection. 
$15 / 26$

\section{Acknowledgement}

No funding regarding this study was received from any sponsors. 


\section{References}

1) Schiller JH, Harrington D, Belani CP, et al. (2002) Comparison of four

chemotherapy regimens for advanced non-small-cell lung cancer. N Engl J Med 346:

92-98

2) de Marinis F, Grossi F (2008) Clinical evidence for second- and third-line treatment options in advanced non-small cell lung cancer. Oncologist 13 Suppl 1:14-20

3) Ellis P, Davies AM, Evans WK, et al. (2006) The use of chemotherapy in patients with advanced malignant pleural mesothelioma: a systematic review and practice guideline. J Thorac Oncol 1:591-601

4) Lara PN Jr. (2000) Malignant thymoma: current status and future directions.

Cancer Treat Rev. 26:127-31

5) Moreno de Vega MJ, Dauden E, Abajo P, et al. (2002) Skin necrosis from extravasation of vinorelbine. J Eur Acad Dermatol Venereol 16:488-90

6) Vescia S, Baumgärtner AK, Jacobs VR, et al (2008) Management of venous port systems in oncology: a review of current evidence. Ann Oncol 19:9-15

7) Kurul S, Saip P, Aydin T (2002) Totally implantable venous-access ports: local problems and extravasation injury. Lancet Oncol 3:684-92.

8) Klotz HP, Schopke W, Kohler A, et al. (1996) Catheter fracture: A rare complication of totally implantable subclavial venous access device. J Surg Oncol 62:225 
9) Povoski SP (2000) A prospective analysis of the cephalic vein cutdown approach for chronic indwelling central venous access in 100 consecutive cancer patients. Ann Surg Oncol 7:496-502

10) Di Carlo I, Barbagallo F, Toro A, et al (2005) External jugular vein cutdown approach, as a useful alternative, supports the choice of the cephalic vein for totally implantable access device placement. Ann Surg Oncol 12:570-3

11) Chang HM, Hsieh $C B$, Hsieh HF, et al (2006) An alternative technique for totally implantable central venous access devices. A retrospective study of 1311 cases. EJSO 32:90-93.

12) Schwarz RE, Groeger JS, Coit DG (1997) Subcutaneously implanted central venous access devices in cancer patients: a prospective analysis. Cancer 79:1635-40

13) Kock HJ, Pietsch M, Krause U, et al. (1998) Implantable vascular access systems: experience in 1500 patients with totally implanted central venous port systems. World J Surg 22:12-16

14) Seiler CM, Frohlich BE, Dorsam UJ et al. (2006) Surgical technique for totally implantable access ports (TIAP) needs improvement: A multivariate analysis of 400 patients. J Surg Oncol 93:24-29

15) Jablon LK, Ugolini KR, Nahmias NC (2006) Cephalic vein cut-down verses 
percutaneous access: a retrospective stydy of complications of implantable venous access devices. Am J Surg 192:63-67

16) Sutherland DE, Weitz IC, Liebman HA (2003) Thromboembolic complications of

Cancer: Epidemiology, pathogenesis, diagnosis, and treatment. Am J Hematol 72:

$43-52$

17) Kuriakose P, Colon-Otero G, Paz-Fumagalli R (2002) Risk of deep venous

thrombosis associated with chest versus arm central venous subcutaneous port

catheters: A 5-year single institution retrospective study. J Vasc Interv Radiol

$13: 179-184$ 
Table 1 Patient Characteristics and Baseline Demographics ( $N=25)$

\begin{tabular}{|c|c|c|}
\hline & & $\begin{array}{l}\text { Number of } \\
\text { Patients }\end{array}$ \\
\hline \multirow[t]{2}{*}{ Sex } & Male & 14 \\
\hline & Female & 11 \\
\hline \multirow[t]{2}{*}{ Age (years) } & Median & 66 \\
\hline & Range & $39-79$ \\
\hline \multirow[t]{3}{*}{ Disease } & Lung cancer & 21 \\
\hline & Malignant pleural mesothelioma & 2 \\
\hline & Invasive thymoma & 2 \\
\hline Purpose of & Chemotherapy & 17 \\
\hline implantation & Chemotherapy with parenteral nutrition & 5 \\
\hline Number of & 0 regimen & 1 \\
\hline Regimens & 1 regimen & 4 \\
\hline \multirow[t]{5}{*}{ before implantation } & 2 regimens & 8 \\
\hline & 3 regimens & 5 \\
\hline & 4 regimens & 5 \\
\hline & 5 regimens & 1 \\
\hline & 11 regimens & 1 \\
\hline Number of & 0 cycle & 1 \\
\hline Cycles & 1 to 5 cycles & 7 \\
\hline \multirow[t]{3}{*}{ before implantation } & 6 to 10 cycles & 7 \\
\hline & 11 to 15 cycles & 5 \\
\hline & 16 or more cycles & 5 \\
\hline
\end{tabular}


Table 2

Complications and problems

\begin{tabular}{lll}
\hline & & Number of \\
& & Patients \\
\hline $\begin{array}{l}\text { Surgical } \\
\text { Complications }\end{array}$ & Hematoma & 1 \\
& & 1 \\
Short-term & Early death without use of port & \\
Complications & Port-site infection caused by bacteremia & 1 \\
& & \\
Long-term & Venous thrombosis & 2 \\
Complications & Skin ulcer & 1 \\
& Port occlusion & 1 \\
\hline
\end{tabular}




\section{Figure Legends}

\section{Figure 1}

After a contrast agent was injected, the direction of the left basilic vein was visualized under radioscopy. Arrowheads indicate the basilic vein.

\section{Figure 2}

A skin incision, approximately $4 \mathrm{~cm}$ long, and in a direction perpendicular to the running of the basilic vein, was made in the center of the ulnar side of the arm and a subcutaneous pocket was prepared for the port body.

\section{Figure 3}

According to the Seldinger method, a 20-gauge indwelling needle was inserted into the basilic vein (3A) under venography $(3 B)$ and a guide wire was introduced through the basilic vein (3C) to the SVC. 


\section{Basilic} vein
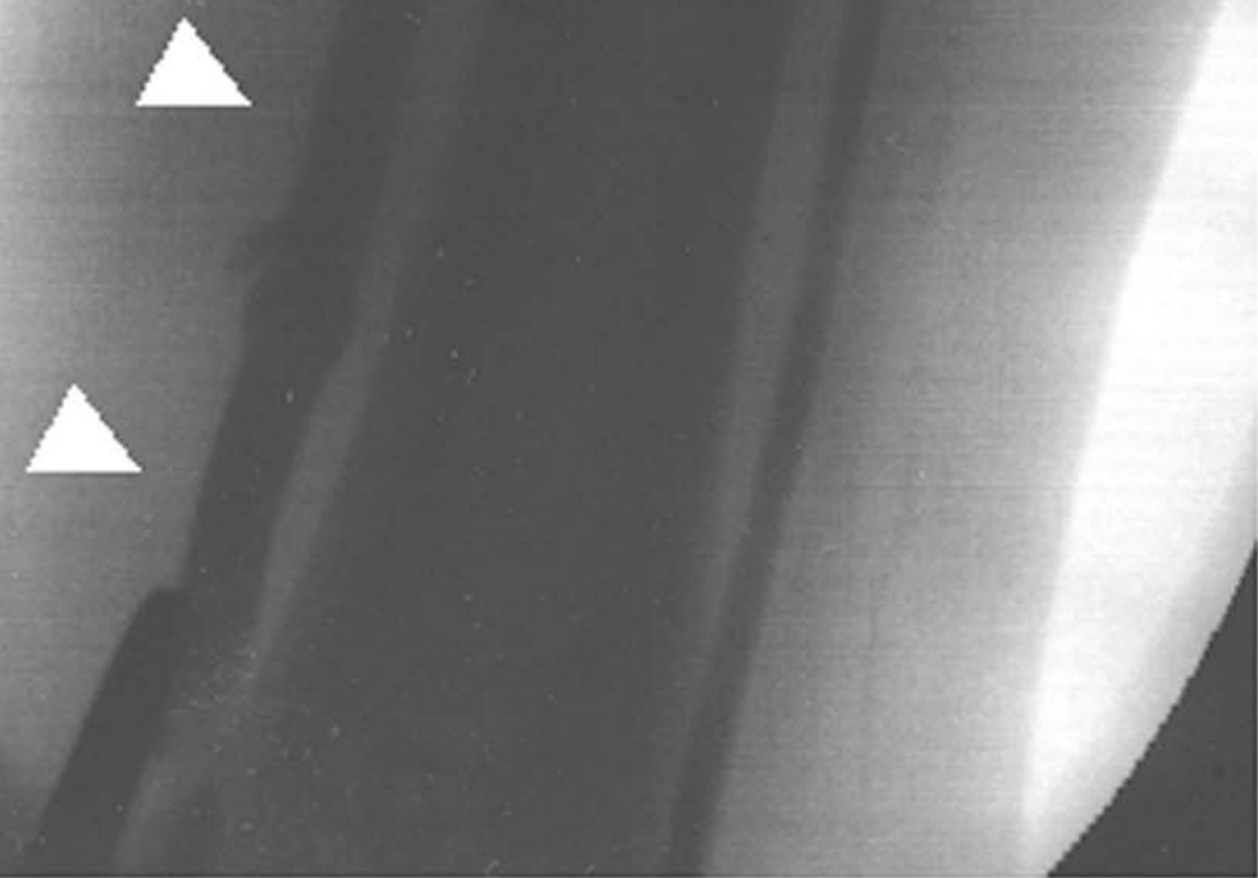

Figure 1 


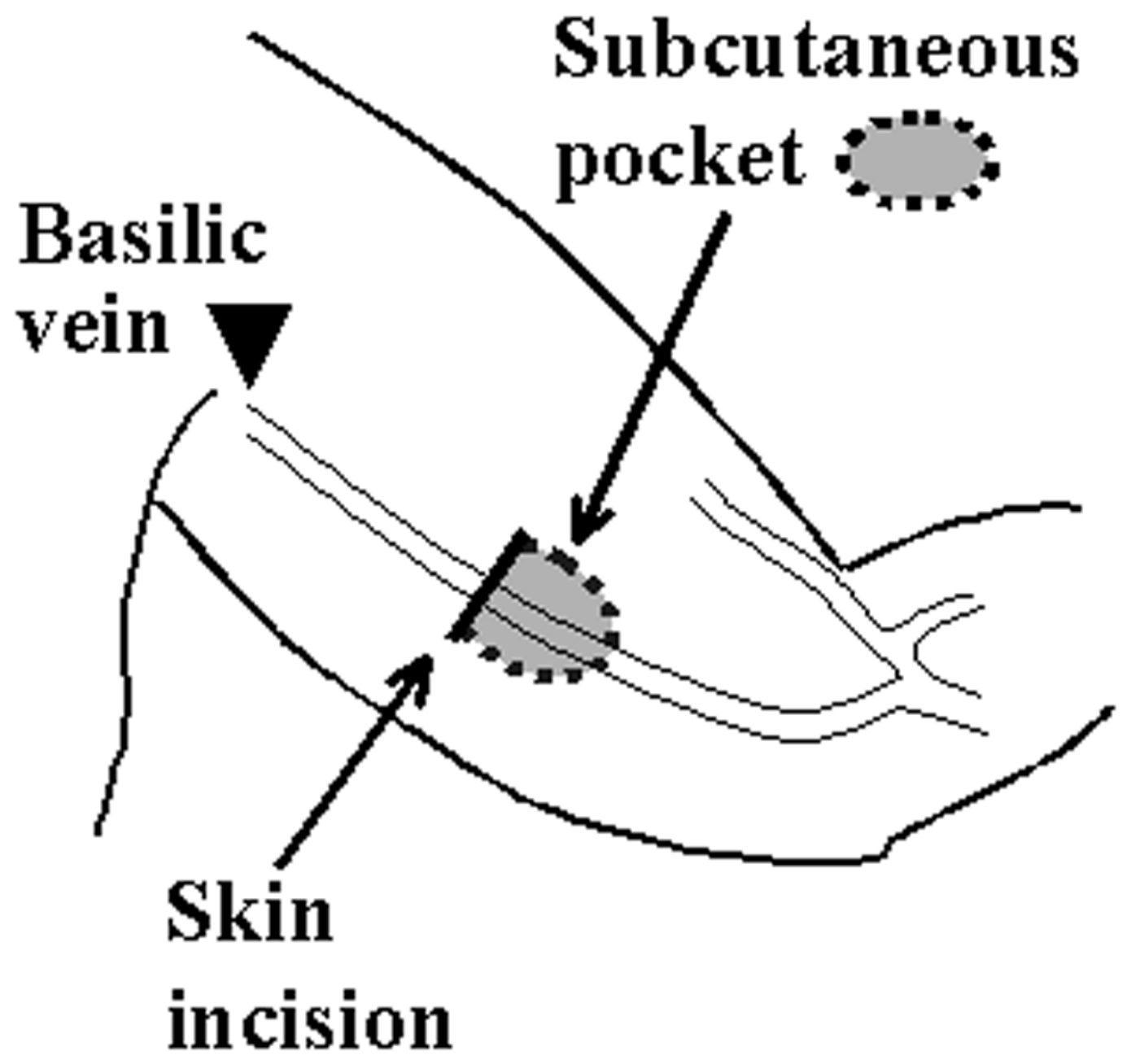

Figure 2 


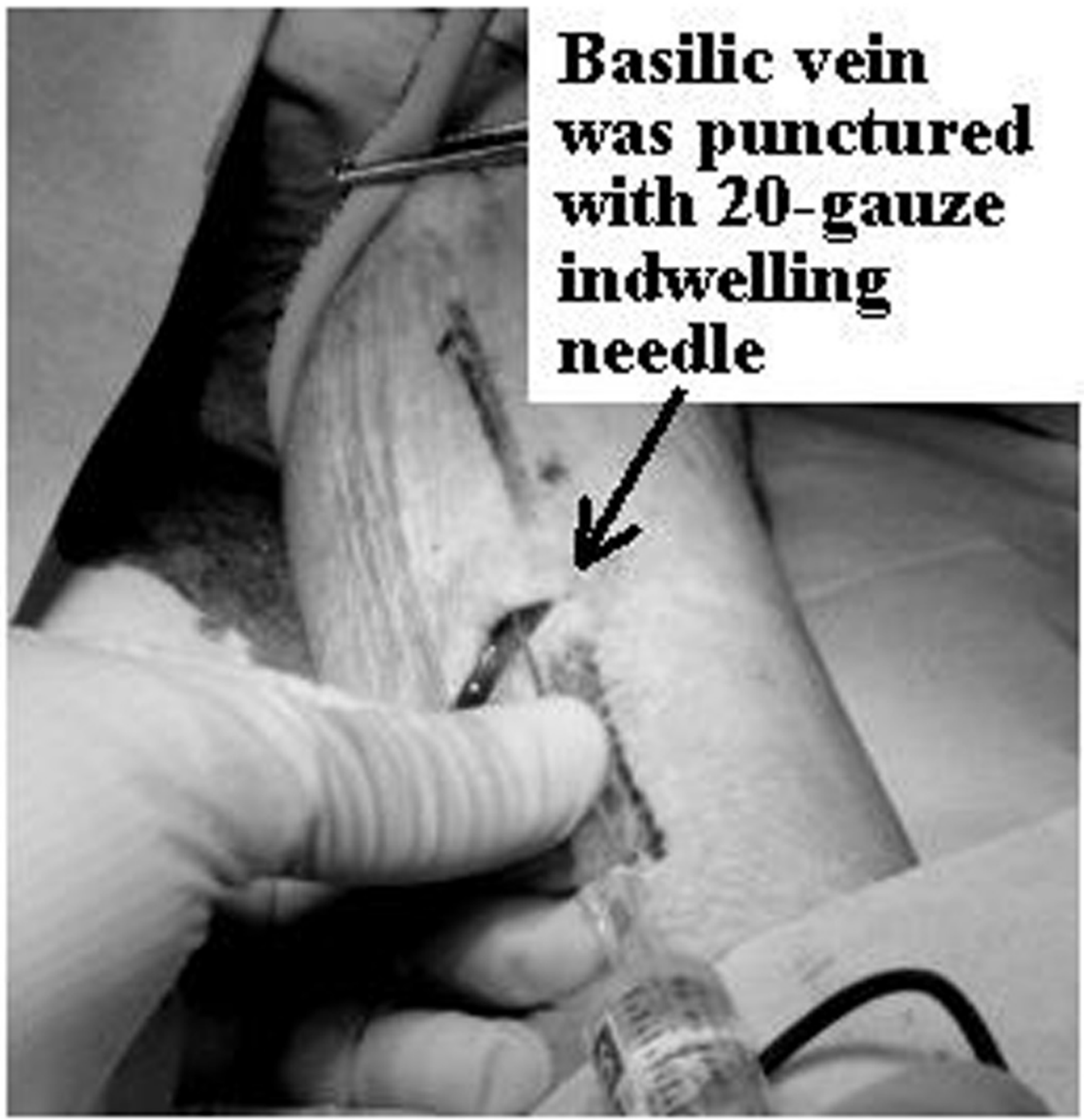

Figure 3A 


\section{Radiographically visualized basilic vein}

\section{Puncture with 20-gauze needle}

Figure 3B 


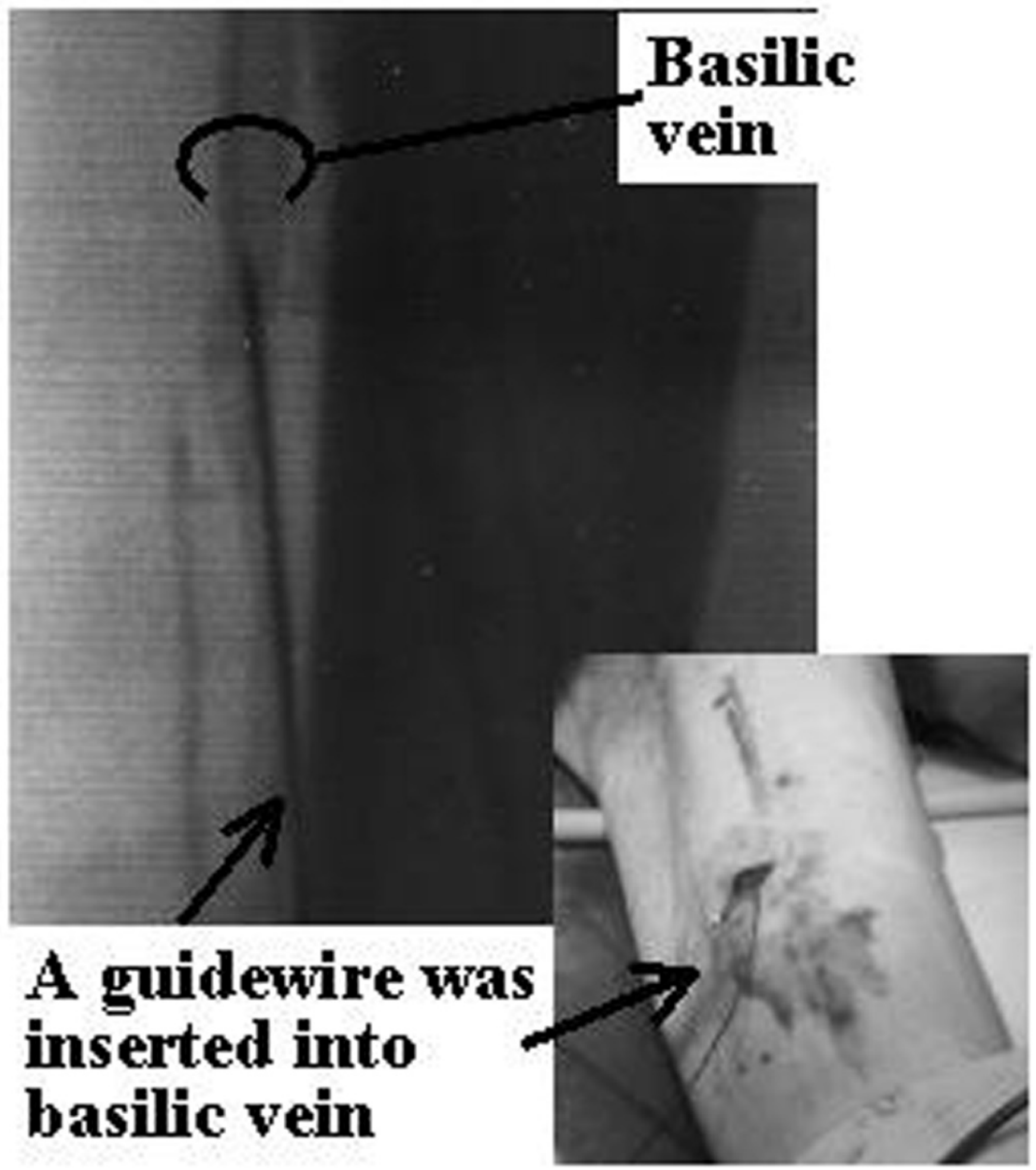

Figure 3C 